\title{
Motricité de l'intestin grêle et digestibilité iléale d'un régime à base de manioc chez le porc
}

\author{
J.-P. LAPLACE et Béatrice DARCY-VRILLON \\ avec la collaboration technique de Georgette Brachet, Brigitte Mourot, \\ Françoise SENE et A. VAN Hecke \\ I.N.R.A., Laboratoire de Physiologie de la Nutrition \\ Centre de Recherches zootechniques, $F 78350$ Jouy-en-Josas
}

\begin{abstract}
Résumé
Quatre porcs mâles castrés de race Large White, d'un poids vif d'environ $50 \mathrm{~kg}$, ont été utilisés pour évaluer les effets de l'incorporation de manioc dans l'aliment, sur la motricité de l'intestin grêle d'une part, et sur la digestibilité apparente à l'extrémité distale de celui-ci, d'autre part. Deux des porcs, équipés d'électrodes chroniques implantées en 7 sites le long de l'intestin grêle, ont permis l'étude de l'organisation de la motricité en complexes myoélectriques migrants lors d'ingestion de régimes semi-purifiés comportant 72,4 p. 100 de farine de manioc ou 68,3 p. 100 d'amidon de maïs purifié, formulés dans les 2 cas à 16 p. 100 de matières azotées totales apportées pour l'essentiel par une farine de poisson. Parallèlement a été testé dans les mêmes conditions expérimentales un régime standard commercial comportant 30 p. 100 de farine de manioc. Les deux autres porcs, préparés par fistulation iléo-colique post-valvulaire, ont permis l'étude cinétique, qualitative et quantitative des digesta recueillis à la jonction iléo-caeco-colique, lors d'ingestion du seul régime semi purifié à base de farine de manioc, comparativement aux résultats obtenus antérieurement pour le régime à base d'amidon de maïs purifié (DARCY, LAPLACE \& Villiers, 1981).

L'ingestion d'un repas quotidien du régime semi-purifié à base de manioc conduit à collecter. à l'extrémité distale de l'intestin grêle, des quantités de matière fraîche et de matière sèche respectivement 2,5 et 1,7 fois plus importantes que pour le régime à base d'amidon de maïs. La teneur en matière sèche des digesta est en moyenne de l'ordre de 7 p. 100 seulement pour le régime manioc contre 11 p. 100 pour le régime maïs. La digestibilité apparente iléale de l'amidon est subtotale pour les 2 régimes alors que celles de la matière sèche et de l'azote sont fortement réduites (environ $78 \mathrm{p}$. 100 pour le régime manioc contre 87 p. 100 pour le régime maïs). L'organisation de la motricité en complexes myoélectriques est globalement préservée avec le régime semi-purifié à base de manioc. Cependant, diverses modifications importantes des caractéristiques des complexes peuvent être directement reliées à l'augmentation du volume de digesta plus fluides en régime manioc. L'élévation du niveau d'ingestion, avec le régime standard, aggrave ces effets, et conduit à une véritable désorganisation de la motricité. Ces observations sont expliquées par la présence dans la farine de manioc de près de 5 p. 100 de sucres simples, susceptibles d'être à l'origine de l'afflux d'eau vers la lumière intestinale et par là même des modifications de la motricité, et par celle de composés phénoliques et tannins sans doute responsables de la moindre digestibilité iléale de la matière sèche et de l'azote. De telles particularités, liées aux caractéristiques qualitatives irrégulières des matières premières, constituent un terrain favorable à l'éclosion d'une pathologie telle que le syndrome de diarrhée récurrente.
\end{abstract}

Mots clés : motricité, intestin grêle, digestibilité iléale, manioc, porc. 


\section{Introduction}

L'évolution relative du coût des matières premières a conduil à une utilisation croissante du manioc en substitution des céréales dans l'alimentation du porc. Mais le manioc disponible sur les marchés est de qualité extrêmement variable tant du fait de sa préparation que de sa conservation. Les problèmes posés par son emploi (présence éventuelle de mycotoxines et d'hétérosides cyanogènes, taux élevés de cellulose et de minéraux, pauvreté en protéines, induction d'un besoin accru en acides aminés soufrés) ont fait l'objet de la revue de OKe (1978). Divers travaux expérimentaux ont tenté d'évaluer les conséquences nutritionnelles et physiopathologiques de l'incorporation de manioc dans les régimes pour porc. Mais il reste à peu près impossible d'aboutir à une conclusion claire, principalement en raison de la diversité des «maniocs» utilisés. Dès 1969 cependant, Aumaıtrı: a rapporté des informations relatives à l'utilisation digestive (digestibilité apparente fécale) et aux performances obtenues avec du manioc comparativement à des céréales, lors d'incorporation aux régimes de sevrage précoce du porcelet. Plus récemment, le travail de Perez et al. (1981) fournit une étude très complète, chez le porc en croissance et le porcelet, des performances zootechniques obtenues avec des maniocs bien caractérisés au plan physicochimique et contrôlés du point de vue sanitaire.

Le présent travail constitue une première approche des effets de l'incorporation du manioc (type Malawi dit Manioc 2 dans l'étude de Prerez et al., 1981), d'une part sur l'organisation de la motricité de l'intestin grêle en complexes myoélectriques migrants (LAPLACE, 1978 a), dont on sait qu'ils déterminent la rapidité du transit dans l'intestin grêle (LAPLACE \& Roman, 1979), et, d'autre part, sur les caractéristiques des contenus digestifs et la digestibilité apparente à l'extrémité distale de l'intestin grêle.

\section{Matériel et méthodes}

\section{A. Animaux et aliments}

Quatre porcs mâles castrés de race Large White ont été utilisés. Deux d'entre eux ont subi, respectivement au poids vif de 50 et $55 \mathrm{~kg}$, une fistulation iléo-colique post-valvulaire (DARCY, LAPLACE \& Villiers, 1980). Les deux autres ont été soumis, à un poids vif de 45,5 et $47 \mathrm{~kg}$ respectivement, à l'implantation d'électrodes souples en 7 sites répartis au long de l'intestin grêle (LAPLACE, 1978 a).

Le régime expérimental dit «manioc» a été formulé à un taux de 16 p. 100 de matières azotées totales par rapport à la matière sèche (M.S.), apportées pour l'essentiel par une farine de poisson d'origine danoise de façon à pouvoir comparer les résultats à ceux antérieurement obtenus avec un aliment à base d'amidon de maïs purifié et de farine de poisson, dit "régime maïs" (DARCY, LAPlaACE \& VilLiers, 1981). Leurs compositions centésimale et chimique sont indiquées dans le tableau 1. Le manioc utilisé pour la fabrication est une farine blutée, préparće à partir de racines de manioc en provenance du Malawi, de la façon suivante : concassage, broyage fin (grilles $1.5 \mathrm{~mm})$, blutage $(3 \times 16$ tamis à mailles de 100$)$, élimination des traces de fer et désinfection à l'oxyde d'éthylène $\left(500 \mathrm{~g} / \mathrm{m}^{*}-72 \mathrm{~h}\right)$. Cette matière première a une teneur en M.S. de 85,2 p. 100 et une teneur en matières azotées totales et amidon (en p. 100 de la M.S.) de 2,6 et 82,3 respectivement. 
DIGESTION DU MANIOC

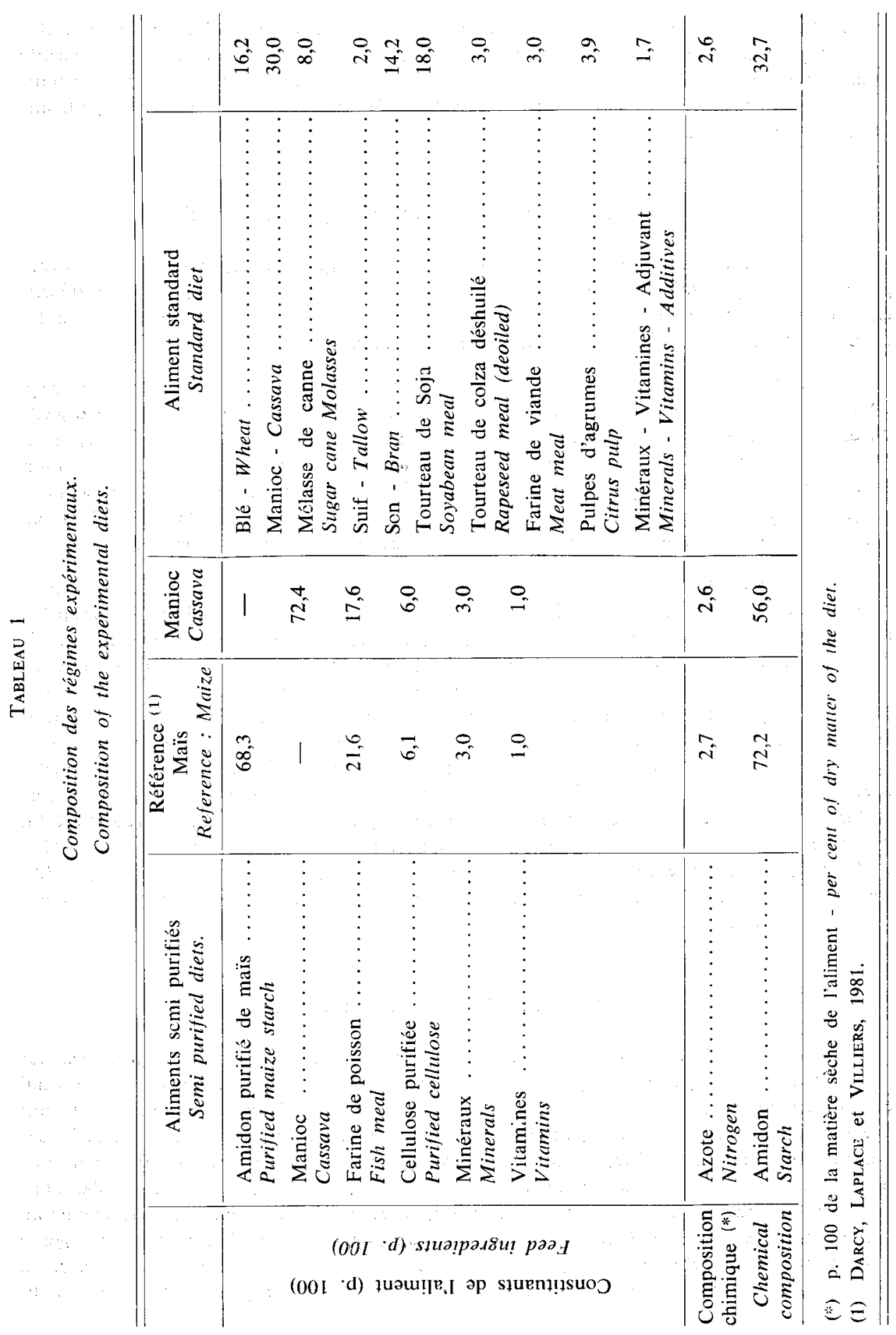


A titre complémentaire, et chez les seuls animaux destinés à l'étude des activités électromyographiques (EMG) de l'intestin grêle, un aliment dit «standard » présenté sous forme de granulé a également été utilisé (tabl. 1). Cet aliment a été prélevé dans les stocks d'un élevage dont un grand nombre d'animaux était atteint du syndrome dit de diarrhée récurrente.

\section{B. Transit dans l'intestin grêle et digestibilité iléale}

Les aspects cinétiques et quantitatifs du passage des digesta dans l'intestin grêle, et la digestibilité apparente à l'extrémité distale de celui-ci, ont été décrits antérieurement pour le régime maïs (DARCY, LAPlace \& Villiers, 1981). Aussi les 2 porcs utilisés ici ont-ils reçu exclusivement l'aliment manioc.

Durant les 10 jours de récupération post-opératoire, les porcs fistulés ont reçu un seul repas quotidien. Puis, chacun des 2 porcs a fait l'objet de collectes de digesta durant 3 nycthémères avec distribution d'un repas unique à $9 \mathrm{~h}$ (ingéré moyen de M.S. : $901 \pm 57 \mathrm{~g}$ ). Ensuite, sans accoutumance préalable, les collectes sont poursuivies durant 2 ou 3 nycthémères avec distribution de deux repas à $9 \mathrm{~h}$ et $17 \mathrm{~h}$ (ingéré moyen quotidien de M.S. : $1423 \pm 80 \mathrm{~g}$ ). Dans tous les cas l'aliment manioc a été distribué sous forme de soupe (farine : 1, eau : 1,5 en poids).

Les digesta ont été collectés selon la technique décrite antérieurement (DARcY, LAPLACE \& Villiers, 1980) soit à 13, 15, 17, 23 et $9 \mathrm{~h}$ le lendemain lorsqu'un seul repas a été distribué, soit à $13,15,17,21,1,5$ et $9 \mathrm{~h}$ le lendemain lors de distribution de 2 repas. Pour chaque collecte, le poids frais total des digesta a été enregistré. Deux échantillons ont été prélevés après homogénéisation. Le premier (environ $20 \mathrm{~g}$ de matière fraîche) a permis de déterminer la quantité de M.S. écoulée au temps post-prandial considéré de façon à décrire la cinétique de passage à la jonction iléo-caeco-colique. Le second (10 p. $100 \mathrm{du}$ poids frais total de la collecte) a été stocké à $+4{ }^{\circ} \mathrm{C}$ et cumulé avec les échantillons homologues obtenus aux divers instants de mesure. Cette quantité cumulée de digesta frais (soit 10 p. 100 du total des digesta de $24 \mathrm{~h}$ ) a été utilisée après lyophilisation pour déterminer la M.S. (24 h à $104^{\circ} \mathrm{C}$ ), l'azote (Kjeldahl) et l'amidon (selon Thivend, Mercier \& Guilbot, 1972). Ces résultats ont ensuite permis de calculer les digestibilités apparentes iléales (CUDa I.) correspondantes.

\section{Activités EMG de l'intestin grêle}

Chacun des 2 porcs équipés d'électrodes intrapariétales souples implantées sur l'intestin grêle a reçu les 3 régimes (maïs, manioc et standard). Pour chaque animal et chaque régime, des enregistrements des activités EMG ont été réalisés durant 3 à 4 nycthémères consécutifs, après accoutumance au régime considéré. Dans le cas des régimes maïs et manioc, les porcs ont systématiquement reçu deux repas par jour à $9 \mathrm{~h}$ et $17 \mathrm{~h}$, sous la forme de soupe (farine : 1, eau : 1,5 en poids). L'aliment commercial granulé a été fourni ad libitum, avec renouvellement et réapprovisionnement tous les jours à $9 \mathrm{~h}$. Dans ces conditions, la quantité d'aliment frais ingérée quotidiennement a été de $2000 \mathrm{~g}$ pour les régimes maïs et manioc ; elle a été d'environ $2400 \mathrm{~g}$ pour le porc 1 et régulièrement de $3000 \mathrm{~g}$ pour le porc 2 dans le cas du régime standard. 
Les activités EMG ont été recueillies sur un polygraphe multivoies (Dynograph RM ou R 611, Beckman), par l'intermédiaire de coupleurs d'entrée (type $9806 \mathrm{~A}$, Beckman) autorisant l'usage de constantes de temps très brèves $(0,03 \mathrm{sec}$.). L'enregistrement direct des activités EMG a été assuré pendant des périodes diurnes de 8 heures $(9 \mathrm{~h}$ à $17 \mathrm{~h})$ à des vitesses de défilement de 2,5 ou $5 \mathrm{~cm} . \mathrm{mn}^{-1}$, simultanément pour les 7 sites de dérivation. Par ailleurs, les activités EMG dérivées en un site proximal et en un site distal de l'intestin grêle ont fait l'objet, $24 \mathrm{~h}$ sur 24, d'une sommation des potentiels d'action (intégration linéaire selon LATOUR, 1973) par pas de $20 \mathrm{sec}$. avec transcription graphique à une vitesse de défilement de $2 \mathrm{~mm}$. sec.-1 (enregistreur potentiométrique CR 552. IJ Instruments).

Au terme des essais prévus, les porcs ont été sacrifiés afin de procéder aussitôt aux mensurations de l'intestin grêle (longueur totale, distance des sites de dérivation par rapport au pylore). Ces mesures permettent de définir la localisation des sites de dérivation des activités EMG sous la forme du pourcentage de la longueur totale de l'intestin grêle que représente la distance qui sépare ce site du pylore. Chez le premier porc, les électrodes ont été localisées à $0,7-4,3-23,0-38,4-55,6-75,3-$ 95,6 p. 100 de la longueur totale $(1153 \mathrm{~cm}$ pour un poids à l'abattage de $67,8 \mathrm{~kg})$; chez le second porc, les électrodes ont été principalement implantées sur la moitié distale, soit à $0,02-29,8-56,4-71,4-79,3-80,7-94,7$ p. 100 de la longueur totale (1505 cm pour un poids à l'abattage de $60,5 \mathrm{~kg})$.

Compte tenu du résultat de ces mesures, les vitesses moyennes de migration des activités régulières ont été estimées sur la base des enregistrements directs simultanés en 7 sites. Leur moyenne harmonique a été calculée respectivement en région proximale ( 0 à $29 \mathrm{p} .100$ ou 0 à $23 \mathrm{p} .100$ selon le porc), médiane ( 29 à 71 p. 100 ou 23 à 75 p. 100$)$ et distale $(71$ à 95 p. 100 ou 75 à 96 p. 100) et pour l'ensemble de l'intestin grêle. Les enregistrements de $24 \mathrm{~h}$ sous forme intégrée ont permis la mesure du nombre de complexes myoélectriques migrants (C.M.M.) par 24 h, de leur durée moyenne, de la durée respective moyenne de chacune de leurs phases constitutives (silence, activité irrégulière, activité régulière). Les valeurs moyennes pour ces divers paramètres sont calculées sur l'ensemble de la période de 24 heures, mais aussi de façon distincte pour les périodes dites diurne (9 à 21 h) et nocturne (21 à 9 h).

\section{Résultats}

\section{A. Passage de la matière fraîche (fig. 1)}

Dans le cas de repas unique, les quantités de matière fraîche recueillies avec le manioc sont toujours plus élevées que celles recueillies avec le maïs, quel que soit le temps post-prandial considéré, alors que les quantités de farine fraîche ingérées sont analogues (soit en moyenne de 1024 et $1035 \mathrm{~g}$ respectivement pour le manioc et le maïs). L'écart entre ces deux régimes s'accroît au fur et à mesure du temps écoulé après le repas. Au total en 24 h, la quantité de digesta frais est de $1090 \mathrm{~g}$ avec le maïs et de $2716 \mathrm{~g}$ avec le manioc, soit 2,5 fois plus pour ce dernier régime. Avec le régime manioc, les quantités de matière sèche recueil- 
lies sont toujours plus élevées lors de distribution sous forme de 2 repas (ingéré moyen de farine fraîche : $1604 \mathrm{~g}$ ) que lors de distribution unique (ingéré moyen de farine fraîche : $1024 \mathrm{~g}$ ). Toutefois, on ne recueille en $24 \mathrm{~h}$ que $3521 \mathrm{~g}$ de digesta frais, soit 1,3 fois ce qui est obtenu lors de distribution d'un repas unique, alors que le rapport des quantités de farine ingérée est de 1,6. Notons enfin que l'écart relatif le plus important est observé à la quatrième heure post-prandiale, où le surcroît de matière fraîche recueillie pourrait correspondre en partie au deuxième repas distribué la veille.

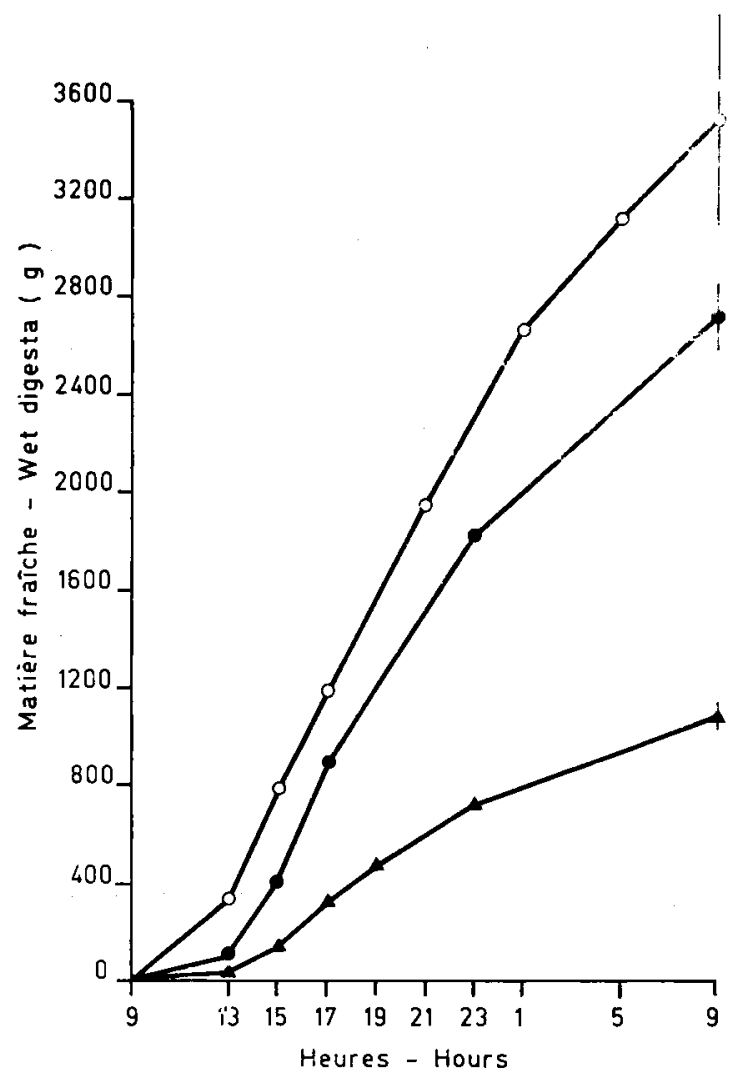

FIG. 1

Quantités moyennes cumulées $(g)$ de matière fraîche collectée à la jonction iléo-caeco-colique en $24 \mathrm{~h}$.

Mean cun:ulated quantities ( $g$ ) of wet digesta collected at the ileo-caeco-colic junction over $24 \mathrm{~h}$.

- Régime manioc, 1 repas par jour : $\mathrm{n}=4$.

Cassava diet, fed once a day : $n=4$.

Régime manioc, 2 repas par jour $: \mathrm{n}=6$.

Cassava diet, fed twice a day : $n=6$.

- Régime maïs, 1 repas par jour. In : Darcy, Laplace \& Villiers, 1981. L'écart type de la moyenne est indiqué pour le cumul en $24 \mathrm{~h}$.

Maize diet, fed once a day. In : Darcy, Laplace, Villiers, 1981. Standard error of the mean is indicated for the cumulated quantity. 


\section{B. Passage de la matière sèche (fig. 2 et 3)}

Des écarts de même sens s'observent en ce qui concerne la M.S. (fig. 2 A). Toutefois, la différence des quantités de digesta secs recueillis en $24 \mathrm{~h}$ est moindre entre manioc (193 g) et maïs (112 g), puisque le rapport n'est que de 1,7 contre 2,5 dans le cas des digesta frais. La quantité de M.S. recueillie en $24 \mathrm{~h}$ est de $290 \mathrm{~g}$ avec deux repas quotidiens de manioc soit 1,5 fois la quantité résiduelle avec un seul repas. Quel que soit le nombre de repas et donc la quantité ingérée au total en 24 h, les quantités relatives de M.S. collectée (en p. 100 de l'ingéré sec) ne diffèrent pas significativement (fig. $2 \mathrm{~B}$ ). Elles sont, par contre, pour le régime manioc, significativement supérieures à celles recueillies pour le régime maìs. La teneur moyenne en M.S. des digesta collectés (fig. 3) diffère significativement d'un

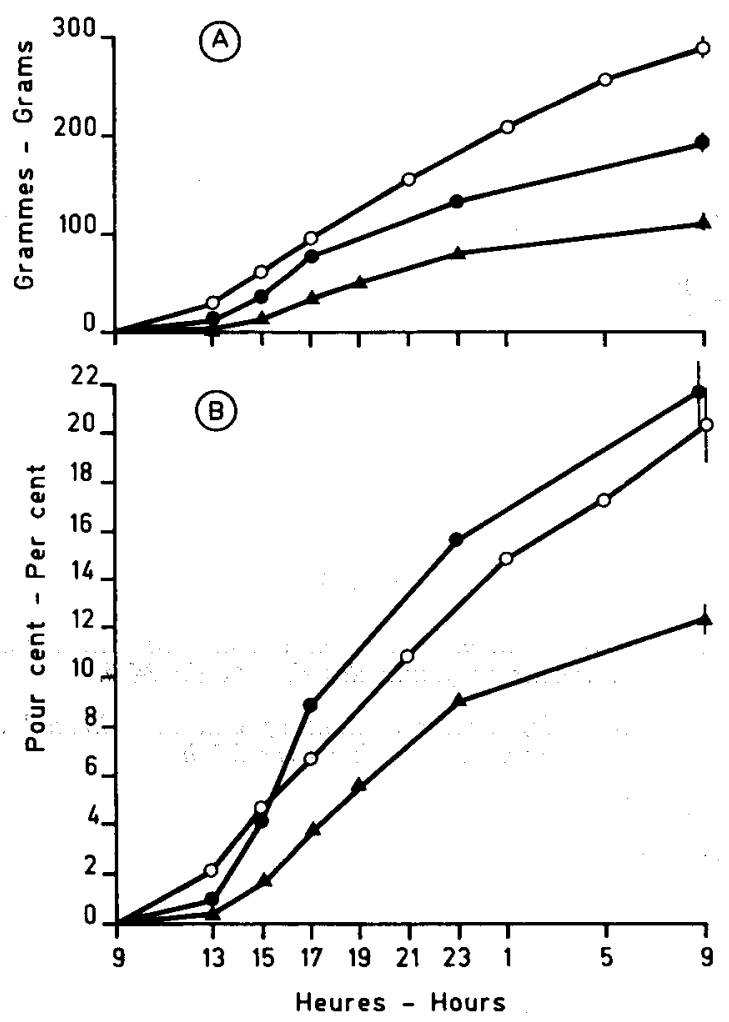

FIG. 2

Quantités moyennes cumulées de matière sèche collectée à la jonction iléo-caeco-colique en 24 h, exprimées en grammes (en haut), et en p. 100 de la quantité de matière sèche ingérée (en bas).

Mean cumulated quantities of dry matter collected at the ileo-caeco-colic junction over $24 h$, expressed as grams (top) and as per cent of the dry matter intake (bottom).

Symboles : voir fig. 1.

Symbols : see fig. 1 . 
régime et d'une situation à l'autre. La hiérarchie observée sur les digesta de $24 \mathrm{~h}$ est vérifiée sur la plupart des collectes du nycthémère. Enfin, alors que la teneur en M.S. augmente significativement au cours du passage des digesta, entre la quatrième et la quatorzième heure pour le régime maïs, elle n'augmente de façon marquée qu'entre la quatrième et la huitième heure pour un repas unique du régime manioc, et reste relativement stable au cours du nycthémère pour deux repas du régime manioc.

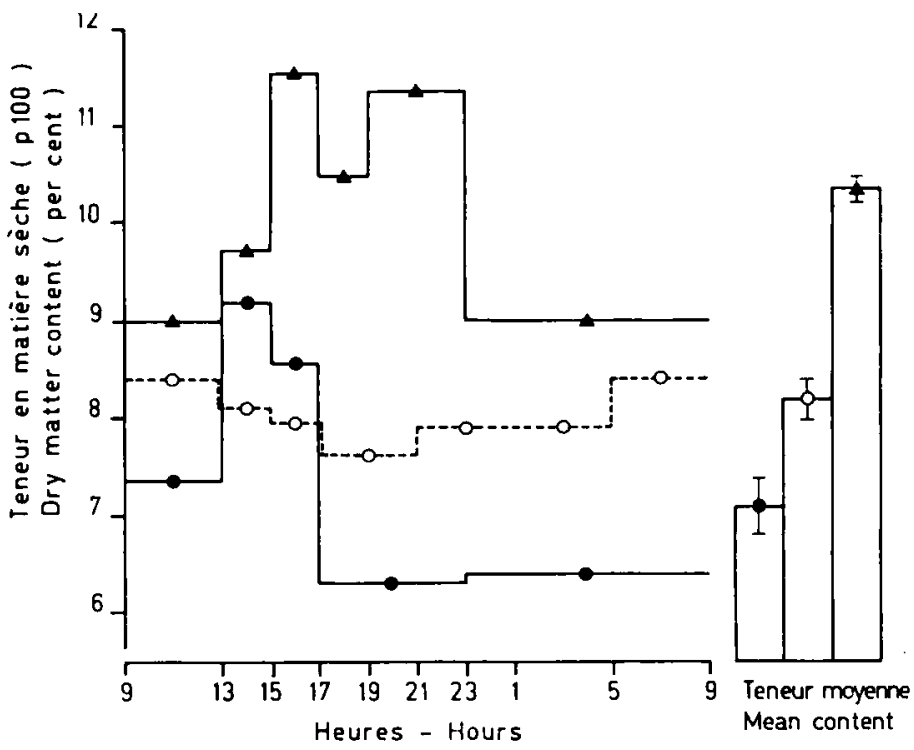

Fig. 3

Evolution de la teneur moyenne en matière sèche des digesta collectés au cours des 24 h, et teneur moyenne des digesta de $24 \mathrm{~h}$.

Evolution of the mean dry matter content of digesta collected during $24 \mathrm{~h}$, and mean value over $24 \mathrm{~h}$.

Symboles : voir fig. 1.

Symbols : see fig. 1 .

C. Digestibilités apparentes (tabl. 2)

Quel que soit le nombre de repas, et donc le niveau d'ingestion, les digestibilités apparentes iléales de la M.S., de l'amidon et de l'azote sont analogues. Elles sont de l'ordre de 78 p. 100 pour la M.S. et pour l'azote, et supérieures à 99 p. 100 pour l'amidon. Si l'on compare l'ensemble des résultats portant sur le manioc à ceux obtenus pour le maïs (DARcy, LAPlace \& Villiers, 1981), on observe dans les deux cas une digestibilité quasi totale de l'amidon. Par contre, les digestibilités apparentes de la M.S. et de l'azote sont significativement plus faibles dans le cas du régime manioc. 


\section{Motricité de l'intestin grêle}

L'examen de l'allure générale des tracés permet à lui seul de constater, lors de distribution des régimes maïs et manioc, que l'organisation de la motricité en C.M.M. est normale (LAPLACE, 1978 a). L'intensité globale des activités EMG, traduite par le nombre et l'amplitude des bouffées de potentiels est dans l'ensemble très modérée ce qui correspond bien au fait que ces deux régimes relativement purifiés sont pauvres en constituants indigestibles susceptibles de stimuler la motricité. Dans le cas de l'aliment standard, on observe également que les activités EMG sont d'une intensité relativement modérée, mais en outre on enregistre une importante modification de l'organisation en C.M.M. par rapport aux aliments purifiés (fig. 4), notamment dans le cas du porc 2 dont le niveau d'ingestion a été régulièrement de $3 \mathrm{~kg}$ par jour.

\section{TABLeaU 2}

Digestibilités iléales apparentes (CUD a I) de la matière sèche (MS) de l'amidon et de l'azote des régimes à base de manioc et d'amidon de maïs.

Apparent ileal digestibilities (CUD a l.) of dry matter (DM) starch and nitrogen of cassava and maïze starch based diets.

\begin{tabular}{|c|c|c|c|}
\hline CUD a I. & $\begin{array}{l}\text { MS } \\
\text { DM }\end{array}$ & $\begin{array}{c}\text { Amidon } \\
\text { Starch }\end{array}$ & $\begin{array}{c}\text { Azote } \\
\text { Nitrogen }\end{array}$ \\
\hline $\begin{array}{l}\text { Manioc ( } 1 \text { repas) .. } \\
\text { Cassava } \\
\text { (I daily meal) }\end{array}$ & $\begin{array}{c}78,30^{\mathrm{a}} \pm 1,38 \\
n=6\end{array}$ & $\begin{array}{c}99,96^{a} \pm 0,008 \\
n=6\end{array}$ & $\begin{array}{c}77,09 a \pm 1,58 \\
n=6\end{array}$ \\
\hline $\begin{array}{l}\text { Manioc (2 repas) } \\
\text { Cassava } \\
\text { (2 daily meals) }\end{array}$ & $\begin{array}{c}79,77^{\mathrm{a}} \pm 0,80 \\
\mathrm{n}=4\end{array}$ & $\begin{array}{c}99,96^{a} \pm 0,003 \\
n=4\end{array}$ & $\begin{array}{c}76,11^{\mathrm{a}} \pm 0,70 \\
\mathrm{n}=4\end{array}$ \\
\hline $\begin{array}{l}\text { Manioc (tous essais } \\
\text { confondus) ....... } \\
\text { Cassava (all trials) }\end{array}$ & $\begin{array}{c}78,89 a \pm 0,88 \\
n=10\end{array}$ & $\begin{array}{c}99,96^{\mathrm{a}} \pm 0,005 \\
\mathrm{n}=10\end{array}$ & $\begin{array}{c}76,70^{a} \pm 0,96 \\
n=10\end{array}$ \\
\hline $\begin{array}{l}\text { Maïs ( } 1 \text { repas) ... } \\
\text { Maize (l daily meal) }\end{array}$ & $\begin{array}{c}87,82^{b} \pm 0,65 \\
n=18\end{array}$ & $\begin{array}{c}99,83^{b} \pm 0,020 \\
n=15\end{array}$ & $\begin{array}{c}86,53^{b} \pm 0,61 \\
n=15\end{array}$ \\
\hline
\end{tabular}

Dans chaque moitié du tableau, les 2 valeurs d'une même colonne affectées de lettres différentes diffèrent au seuil $\mathrm{p}<0,01$ (t de Student).

In each half of the table, the 2 values in a column bearing different letters differ according to Student's $t$ test $(p<0.01)$. 

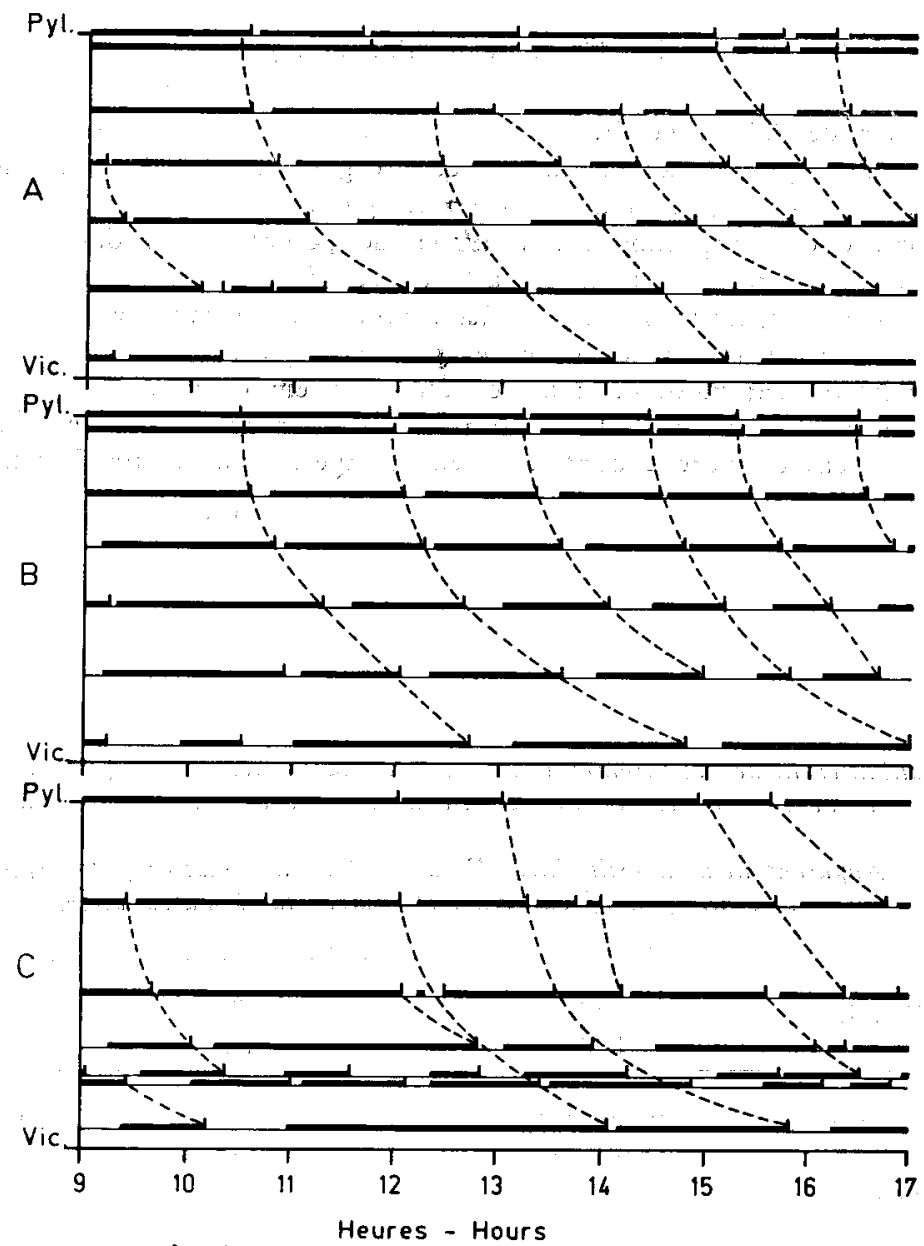

Fig. 4

Représentation schématique de l'organisation en C.M.M.

au cours des $8 \mathrm{~h}$ consécutives au repas du matin, selon la nature de l'aliment.

Schematic picture of the M.M.C. pattern over $8 \mathrm{~h}$ following the morning meal according to the diet.

En $A$, porc 1, régime maïs; en $B$, porc 1 , régime manioc; en $C$, porc 2 , régime standard (3 kg par jour). Les lignes successives correspondent chacune à un site d'enregistrement, repéré par isa localisation proximo distale en pourcentage de la longueur pylore (Pyl.) - valvule iléo-caecale (Vic.). Sur chaque ligne, les barrettes horizontales représentent les épisodes d'activité irrégulière (phase II des C.M.M.) et les barrettes verticales indiquent les activités régulières (phase III). Les lignes pointillées indiquent la migration proximo distale de ces dernières.

$A$, pig 1, maize diet; $B$, pig 1, cassava diet; $C$, pig 2 , standard diet ( $3 \mathrm{~kg}$ per day). Each line of graphs corresponds to a recording site, identified by its distance to the pylorus as a percent of total small bowel length. Along each line the horizontal bars show the irregular spiking activity phases (phase II of MMC); the vertical bars show the regular spiking activity phases (phase III of MMC), whose proximo distal migration is shown by the dotted lines. 
Le dénombrement des activités régulières en $24 \mathrm{~h}$ (tabl. 3), fournit une estimation du nombre total de C.M.M. Les valeurs enregistrées en région proximale de l'intestin grêle, pour les trois régimes à des niveaux d'ingestion n'excédant pas $2,4 \mathrm{~kg}$ par jour, se situent dans la plage des valeurs de référence pour des régimes standard ou semi-purifiés (LAPLACE, 1978 a), soit 17 à 22 C.M.M. par 24 h. Par contre, en région distale de l'intestin grêle, pour le même niveau d'ingestion des trois régimes étudiés, on enregistre des valeurs sensiblement plus élevées, soit 16 à 18 C.M.M. par $24 \mathrm{~h}$ (valeurs de référence 13 à 14), ce qui traduit un pourcentage de disparition des activités régulières, avant achèvement de leur migration proximo-distale complète, de l'ordre de 15 p. 100 au lieu de 30 à 40 p. 100 habituels (LAPLACE, 1978 a). Enfin, lors d'ingestion du régime standard à raison de $3 \mathrm{~kg}$ par jour, on ne dénombre que 14 à 16 activités régulières en région proximale, mais environ 20 par $24 \mathrm{~h}$ en région distale. L'étude des enregistrements directs chez le porc concerné, pour lequel on dispose de 4 sites d'électrodes sur le quart distal de l'intestin grêle, montre que cette particularité tient à l'apparition sur les segments distaux d'activités régulières surnuméraires, c'est-à-dire ne constituant pas l'aboutissement d'une migration proximo-distale complète au long de l'organe. La mesure de la période des activités régulières (tabl. 3) qui indique la durée moyenne des C.M.M. en un site donné, cohérente avec les dénombrements ci-dessus, fournit pour les 3 régimes (2,4 kg par jour au plus) et quel que soit le site intestinal, des valeurs de l'ordre de 70 à $80 \mathrm{mn}$. Lors d'ingestion du régime standard à raison de $3 \mathrm{~kg}$ par jour, la période est en moyenne de $92 \mathrm{mn}$ en région proximale, mais seulement de $71 \mathrm{mn}$ en région distale. Le calcul de la durée moyenne des C.M.M. respectivement pour les périodes dites diurnes et nocturnes ne révèle pas de différence notable par rapport aux indications précédentes.

\section{TABLeau 3}

Nombre total de CMM par période de $24 \mathrm{~h}$, et période moyenne de récurrence des activités régulic̀res au cours du nycthémère en dehors des périodes postprandiales sur les segments proximaux $(P)$ et distaux $(D)$ de l'intestin grêle.

Total number of myoelectric migrating complexes (MMC) per $24 \mathrm{~h}$, and mean period of recurrence of regular activities over $24 h$ (postprandial pattern excepted) in the proximal $(P)$ and distal $(D)$ small intestine.

\begin{tabular}{|c|c|c|c|c|}
\hline $\begin{array}{l}\text { Régime } \\
\text { Diet }\end{array}$ & $\begin{array}{l}\text { Maïs } \\
\text { Maize }\end{array}$ & $\begin{array}{l}\text { Manioc } \\
\text { Cassava }\end{array}$ & \multicolumn{2}{|c|}{$\begin{array}{l}\text { Standard } \\
\text { Standard }\end{array}$} \\
\hline $\begin{array}{l}\text { Porc } \\
\text { Pig }\end{array}$ & 1 & 1 & 1 & 2 (a) \\
\hline $\begin{array}{l}\text { Nombre de CMM en } 24 \mathrm{~h} \ldots \ldots \ldots \\
\text { Number of } M M C \text { over } 24 \text { hrs }\end{array}$ & $\begin{array}{ll}\mathrm{P} & : 17-21 \\
\mathrm{D} & : 16-18 \\
\end{array}$ & $\begin{array}{l}19-21 \\
16-18\end{array}$ & $\begin{array}{l}20-22 \\
15-18 \\
\end{array}$ & $\begin{array}{l}14-16 \\
19-20\end{array}$ \\
\hline $\begin{array}{l}\text { Période moyenne } \ldots \ldots \ldots \ldots \\
\text { ( } \pm \text { écart type de la moyenne) }\end{array}$ & $\begin{array}{r}P: 76,6 \\
\pm 5,0\end{array}$ & $\begin{array}{r}71,7 \\
+\quad 3,0\end{array}$ & $\begin{array}{r}64,6 \\
\pm \quad 3,1\end{array}$ & $\begin{array}{r}92,4 \\
\pm \quad 6,2\end{array}$ \\
\hline $\begin{array}{l}\text { Mean period } \\
( \pm \text { s.e.m. })\end{array}$ & 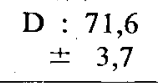 & $\begin{array}{r}83,8 \\
\pm \quad 4,7 \\
\end{array}$ & $\begin{array}{r}82,9 \\
\pm \quad 4,5\end{array}$ & $\begin{array}{r}71,5 \\
\pm \quad 6,7\end{array}$ \\
\hline
\end{tabular}

Données fondées sur 3 nycthémères d'enregistrement - Data based on $3-24 \mathrm{~h}$ records.

(a) Ingestion : $3 \mathrm{~kg}$ par jour - Intake : $3 \mathrm{~kg}$ per day. 
La mesure de la durée respective de chacune des 3 phases qui constituent les C.M.M. apporte quelques éléments supplémentaires. Quels que soient le régime ingéré ou le niveau d'ingestion, le porc concerné ou la période étudiée (diurne ou nocturne), la durée moyenne des épisodes d'activité régulière est très stable, toutes les valeurs étant comprises entre 3 et $4 \mathrm{mn}$, soit des chiffres usuels. Par contre, il existe entre les 3 régimes, pour un même niveau d'ingestion limité à $2 \mathrm{~kg}$ par jour, des différences dans la durée des phases d'activité irrégulière, et notamment de l'évolution de cette durée entre les sites proximaux et les sites distaux de l'intestin grêle. Ces différences coïncident avec des variations de sens inverse de la durée des épisodes de quiescence, à l'intérieur de la durée stable (70-80 mn) des C.M.M.

Tableau 4

Durée moyenne (mn) des phases dactivité irrégulière des CMM (avec écart-type de la moyenne) selon le régime,

sur les segments proximaux $(P)$ et distaux $(D)$ de l'intestin grêle.

Mean duration (min.) of the irregular activity phases of the MMC $( \pm$ S.E.M.) according to the diet, in the proximal $(P)$ and distal $(D)$ small intestine.

\begin{tabular}{l|c|c|c|c|c}
\hline $\begin{array}{c}\text { Période } \\
\text { de mesure } \\
\text { Recording } \\
\text { period }\end{array}$ & $\begin{array}{c}\text { Régime } \\
\text { Diet } \\
\text { Porc } \\
\text { Pig }\end{array}$ & $\begin{array}{c}\text { Maïs } \\
\text { Maize }\end{array}$ & 1 & $\begin{array}{c}\text { Manioc } \\
\text { Cassava }\end{array}$ & \multicolumn{2}{|c}{$\begin{array}{c}\text { Standard } \\
\text { Standard }\end{array}$} \\
\hline $\begin{array}{l}\text { Diurne } \\
(9 \mathrm{~h}-21 \mathrm{~h})\end{array}$ & $\mathrm{P}$ & $84,44 \pm 8,26$ & $67,50 \pm 4,86$ & $58,91 \pm 4,57$ & $86,31 \pm 8,46$ \\
$\begin{array}{l}\text { Daylight } \\
(9 \text { am }-9 \mathrm{pm})\end{array}$ & $\mathrm{D}$ & $47,38 \pm 6,38$ & $67,47 \pm 8,14$ & $46,67 \pm 3,98$ & $36,55 \pm 7,00$ \\
\hline $\begin{array}{l}\text { Nocturne } \\
(21 \mathrm{~h} \text { à } 9 \mathrm{~h})\end{array}$ & $\mathrm{P}$ & $44,00 \pm 3,24$ & $55,60 \pm 4,59$ & $48,91 \pm 3,44$ & $73,14 \pm 11,46$ \\
$\begin{array}{l}\text { Darkness } \\
(9 \text { pm }-9 \text { am })\end{array}$ & $\mathrm{D}$ & $27,42 \pm 3,44$ & $55,20 \pm 6,18$ & $44,08 \pm 8,56$ & $36,44 \pm 8,76$ \\
\hline
\end{tabular}

Données fondées sur 3 nycthémères d'enregistrement - Data based on 3 - 24 h records.

(a) Ingestion : $3 \mathrm{~kg}$ par jour - Intake : $3 \mathrm{~kg}$ per day.

Ainsi, pour le régime maïs on retrouve (tabl. 4) les phénomènes normaux (LAPLACE, 1978 a) . (i) réduction de la durée moyenne des activités irrégulières en période nocturne par rapport à la période diurne, du fait de la plus grande vacuité du tube digestif, et ce tant en région proximale que distale; (ii) réduction également de leur durée moyenne sur les sites distaux par rapport à l'intestin grêle proximal, tant en période diurne que nocturne, du fait de l'absorption qui diminue le volume de digesta en transit. Par rapport à cette situation, on observe lors d'ingestion du 
régime manioc que la durée moyenne des activités irrégulières est réduite en période nocturne, quel que soit le site intestinal concerné ; cette réduction d'environ 18 p. 100 est cependant de moindre ampleur que pour le régime maïs (42 à 48 p. 100). De plus, tant en période diurne que nocturne, il n'y a pas pour le régime manioc de réduction de la durée des activités irrégulières entre les sites proximaux et distaux. Dans le cas du régime standard, la durée moyenne des activités irrégulières est également réduite en période nocturne, d'environ 17 p. 100 pour les sites proximaux mais 5 p. 100 seulement pour les sites distaux. Il existe pour ce régime une certaine réduction de leur durée entre intestin proximal et distal, mais d'ampleur beaucoup plus faible que dans le cas du régime maïs de référence $(-21$ contre -44 p. 100 en période diurne). Enfin, dans le cas de ce même régime standard, pour un niveau d'ingestion de $3 \mathrm{~kg}$ par jour, la différence des durées moyennes d'activité irrégulière entre périodes diurne et nocturne est faible ou nulle, ce qui est cohérent avec l'étalement des repas sur les 24 heures en situation ad libitum. D'autre part, il existe une réduction de 50 à 58 p. 100 de la durée moyenne des activités irrégulières en région distale par rapport à l'intestin proximal, mais ce phénomène résulte pour partie du raccourcissement (d'environ 23 p. 100) de la durée moyenne des C.M.M.

\section{TABLEAU 5}

Vitesses moyennes de migration (moyennes harmoniques, en $\mathrm{cm} . \mathrm{mn}^{-1}$ ) des activités régulières sur les segments proximaux $(P)$, médians (M) et distaux (D) de l'intestin grêle et vitesse moyenne sur l'ensemble du viscère avec indication de la durée estimée de migration proximodistale complète compte tenu de la longueur mesurée de l'intestin de chaque porc.

Mean migration velocity of the regular spiking activities (harmonic mean, $c m . m i n-1$ ) over the proximal $(P)$, middle $(M)$ and distal $(D)$ small intestine,

and mean velocity over the whole small intestine with estimated duration for a full proximo distal migration, taking into account the measured tract length in each pig.

\begin{tabular}{|c|c|c|c|c|c|}
\hline \multirow{2}{*}{ 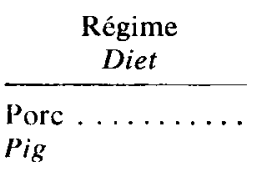 } & \multicolumn{2}{|c|}{$\begin{array}{c}\text { Maïs } \\
\text { Maize }\end{array}$} & \multicolumn{2}{|c|}{$\begin{array}{l}\text { Manioc } \\
\text { Cassava }\end{array}$} & \multirow{2}{*}{$\begin{array}{c}\text { Standard } \\
\text { Standard } \\
2 \text { (a) }\end{array}$} \\
\hline & 1 & 2 & 1 & 2 & \\
\hline $\mathrm{P}$ & 31,95 & 62,25 & 27,56 & 52,27 & 57,57 \\
\hline$M$ & 8,68 & 15,04 & 7,18 & 13,10 & 13,02 \\
\hline D & 5,81 & 6,02 & 3,96 & 2,04 & 1,56 \\
\hline $\begin{array}{l}\text { Grêle total } \ldots . \\
\text { Whole small } \\
\text { intestine }\end{array}$ & $\begin{array}{c}11,95 \\
(96 \mathrm{mn})\end{array}$ & $\begin{array}{c}12,12 \\
(124 \mathrm{mn})\end{array}$ & $\begin{array}{c}8,94 \\
(129 \mathrm{mn})\end{array}$ & $\begin{array}{c}4,83 \\
(312 m n)\end{array}$ & $\begin{array}{c}3,23 \\
(466 \mathrm{mn})\end{array}$ \\
\hline
\end{tabular}

(a) Ingestion : $3 \mathrm{~kg}$ par jour - Intake : $3 \mathrm{~kg}$ per day. 
L'étude des enregistrements simultanés en 7 sites intestinaux, permet d'estimer la vitesse de migration proximo-distale des activités régulières, et de calculer la vitesse moyenne harmonique de migration pour chaque porc et chaque régime. Au-delà de l'existence connue d'une décroissance proximo-distale de la vitesse de migration et de l'importante variabilité de ce critère entre individus (LAPLACE, 1978 a), particularités vérifiées pour les vitesses enregistrées ici (tabl. 5) il est un fait remarquable : à tous les étages de l'intestin grêle, les vitesses moyennes de migration sont plus faibles pour les régimes manioc et standard que pour le régime maïs. Ce phénomène est particulièrement net sur l'intestin grêle distal. Il en résulte qu'au total la vitesse moyenne de migration (du pylore à la jonction iléo-caeco-colique) est approximativement deux fois plus faible pour les régimes comportant du manioc. Le temps total nécessaire à une migration proximo-distale complète est donc considérablement augmenté, même si la valeur obtenue chez le porc 2 à fort niveau d'ingestion doit être considérée avec des réserves en raison du nombre restreint de migrations complètes du fait de la désorganisation du profil moteur de l'intestin dans cette situation.

\section{Discussion}

Les observations relatives à la motricité de l'intestin grêle suggèrent que l'introduction du manioc dans l'alimentation du porc est susceptible d'affecter l'organisation des activités EMG de façon non négligeable, à la limite de la déviation pathologique. L'interprétation des particularités enregistrées paraît cependant relativement aisée à la lumière des caractéristiques des digesta recueillis à la jonction iléo-caeco-colique (quantités de contenus frais et teneurs en M.S., selon le régime).

Si la faible intensité globale des activités EMG est normale pour les régimes semi-purifiés maïs ou manioc, il est a priori surprenant qu'elle reste modeste pour le régime standard qui comporte, entre autres sources de constituants pariétaux, 14,2 p. 100 de son. La substitution de manioc au maîs dans l'aliment purifié s'accompagne d'une teneur en M.S. plus faible (i.e. une plus grande hydratation) des digesta. Un effet similaire dans le cas de l'aliment standard, quoique de moindre amplitude compte tenu de la proportion plus faible de manioc dans cet aliment, peut être responsable de cette particularité. La coexistence d'activités EMG de faible intensité et d'un débit important de digesta très liquides a en effet été soulignée antérieurement après ingestion d'un régime riche en lactose qui induit un afflux d'eau vers la lumière (LAPLACE, 1978 b). Une situation analogue, quoique moins drastique, paraît en cause ici.

Plus remarquable encore est l'importante perturbation de l'organisation en C.M.M. des activités EMG de l'intestin grêle dans le cas du régime standard. Ce désordre a, a priori, d'autant moins de raison d'être que cet aliment a été consommé ad libitum, situation qui autorise le fractionnement de la ration quotidienne en plusieurs petits repas spontanés, et de ce fait conduit à l'absence des épisodes d'activité post-prandiale continue qui masquent les C.M.M. durant 2 à $3 \mathrm{~h}$ après un repas important (RucKEBUSCH \& Bueno, 1976 ; LAPLACE, 1978 a). Mais ici deux effets semblent se conjuguer : l'un tient à l'augmentation de la masse de contenus frais, liée à la présence d'une plus grande quantité d'eau induite par le manioc dans la lumière; l'autre résulte du 
niveau d'ingestion élevé de cet aliment standard, surtout chez le porc 2 pour lequel la désorganisation est la plus spectaculaire.

Pour ces deux raisons, le volume des digesta présents dans l'intestin grêle est probablement tel que se trouve atteint le seuil au-delà duquel apparaissent les modifications des C.M.M. On sait en effet que selon le degré de l'augmentation du volume des digesta, il peut y avoir suspension temporaire des C.M.M. (cas de l'organisation post-prandiale à la suite d'un repas unique volumineux chez le porc ou le chien), renforcement des C.M.M. avec migration proximo-distale complète systématique d'un plus grand nombre ou de toutes les activités régulières et apparition d'activités régulières surnuméraires, et enfin désorganisation totale avec une forte activité EMG échappant à toute systématisation. Ces phénomènes qui marquent une frontière graduée du fonctionnement normal vers le désordre pathologique, et qui peuvent être induits expérimentalement, sont connus aussi bien chez le chien et le mouton (Bueno \& Ruckebusch, 1978) que chez le porc (LAPlace, 1980). Ainsi la surcharge expérimentale par perfusion de digesta additionnels chez le porc alimenté conduit à une suspension plus ou moins prononcée de la périodicité des C.M.M., avec prolongation des épisodes d'activité irrégulière dont l'intensité est de surcroît réduite, et avec ralentissement de la migration des activités régulières (LAPLACE, 1980).

De fait, la plupart de ces modifications connues sont retrouvées dans le présent travail. Pour le régime semi-purifié à 72 p. 100 de manioc, ou pour le régime standard à 30 p. 100 de manioc, ingéré en quantité modérée, a été notée la réduction du taux de disparition des activités régulières avant migration complète. Lors d'ingestion déjà excessive du régime standard, il y a tout à la fois réduction du nombre de C.M.M. avec prolongation d'une activité irrégulière d'intensité modérée sur l'intestin grêle proximal, et renforcement avec apparition d'activités régulières surnuméraires sur l'intestin grêle distal, cependant que la migration proximo-distale est globalement très perturbée. Il s'ajoute à ce tableau le ralentissement considérable de la vitesse de migration des C.M.M., tant pour le régime manioc que pour le régime standard. D’évidence, toutes les particularités de la motricité intestinale qui ont été soulignées relèvent d'un volume excessif de digesta dans la lumière. Elles sont à rapprocher des effets sur les contenus digestifs : (i) la substitution du manioc au maïs pour 70 p. 100 d'un régime semi-purifié conduit, à ingéré égal et pour un repas quotidien unique, à multiplier par 2,5 la quantité de matière frâiche collectée en $24 \mathrm{~h}$ à la jonction iléo-caeco-colique; (ii) pour la même situation, le manioc conduit à un abaissement de plus de 3 p. 100 de la teneur en M.S. des digesta collectés.

Enfin, de façon complémentaire, il existe une relation entre certaines caractéristiques des activités irrégulières et la cinétique de passage des digesta. Alors qu'il y a pour le régime maîs une vague de transit des digesta du repas très nette entre 4 et $14 \mathrm{~h}$ après le repas, en accord avec les résultats antérieurs (DARCY, LAPLACE \& Villiers, 1980-1981), on n'observe qu'une brève vague décelable pour le régime manioc entre 4 et $8 \mathrm{~h}$ après le repas unique, voire pas de variation notable (manioc, 2 repas). Ceci implique donc pour le régime manioc un débit de digesta relativement stable, donc excessif de façon soutenue et non temporaire. Compte tenu de la relation privilégiée entre activité irrégulière et transit des digesta (LAPLACE \& RomAN, 1979), il n'est pas surprenant dans ces conditions que la durée des activités irrégulières ne soit pas sensiblement réduite en période nocturne lors d'ingestion du régime manioc. De plus ce débit important et quasi permanent affecte sans nul doute la 
totalité de l'intestin grêle, comme en témoignent les désordres de la motricité en région distale et l'absence de réduction de la durée des activités irrégulières en ces sites distaux par rapport à l'intestin grêle proximal.

La question se trouve donc posée de l'origine de ce débit de digesta excessif qui paraît être la cause directe des modifications de la motricité de l'intestin grêle. D'évidence la présence de manioc en substitution du maïs dans l'aliment a deux conséquences : (i) une moindre digestion de la matière sèche, la quantité recueillie en $24 \mathrm{~h}$ étant 1,7 fois plus élevée à ingéré égal d'un repas unique du régime manioc par rapport au maïs; (ii) une plus grande quantité d'eau dans les digesta, attestée par leur moindre teneur en matière sèche, sans doute liée à un afflux vers la lumière du fait de la présence de certains constituants du manioc peu ou pas digérés dans l'intestin grêle. Ceci implique très certainement une différence quantitative et qualitative de la digestion du manioc dans l'intestin grêle relativement au maïs. Les CUDaI montrent que, d'une part la digestibilité de l'amidon est subtotale pour les deux régimes, et d'autre part que les digestibilités de la matière sèche et de l'azote dans l'intestin grêle sont d'environ 10 points plus faibles pour le régime manioc par rapport au mais. Cette particularité, dans le cas de l'azote, n'est pas explicable par la seule présence des protéines apportées par la farine de manioc qui représentent 11 p. 100 des protéines totales du régime et dont on ignore la digestibilité.

En dehors de toute interférence microbienne ou virale, il faut s'interroger sur les facteurs nutritionnels susceptibles d'expliquer la baisse de la digestibilité iléale de la matière sèche et de l'azote, et de provoquer un afflux d'eau vers la lumière. La digestibilité quasi totale de l'amidon ne doit pas faire illusion. S'il n'y a pas d'ambiguïté pour le régime maïs dont les glucides sont essentiellement apportés par un amidon purifié, il n'en va pas de même pour le régime manioc. Dans ce cas, la source d'amidon est une farine blutée, qui apporte, en p. 100 de la M.S. de cette matière première, 82,3 p. 100 d'amidon, 2,6 p. 100 de matières azotées, et quelques 4 p. 100 au total de minéraux et cellulose brute. Par conséquent, 11 p. 100 de la M.S. de la farine de manioc sont constitués par des produits autres que ceux énumérés ci-dessus. Il apparaît effectivement que 4,7 p. 100 de la M.S. de la farine de manioc (matière première) sont constitués par des glucides alcoolo-solubles, soit : fructose 0,3 ; glucose 0,5 ; saccharose 3,4 ; maltose 0,1 ; maltotriose 0,1 ; maltotétraose 0,4 (1). Cette proportion non négligeable de glucides simples dans la farine de manioc utilisée, sans doute imputable à un début de dégradation de l'amidon au cours d'une préparation ou conservation imparfaite, est susceptible d'expliquer un afflux osmotique d'eau vers la lumière digestive. Enfin, la fraction résiduelle non identifiée des constituants de cette matière première pourrait comporter essentiellement des composés phénoliques et des tannins. En effet, la farine de manioc utilisée, de teinte légèrement brune, ne peut qu'être une farine de racines non ou mal épluchées. La présence de composés phénoliques et tannins pourrait donc être un facteur explicatif de la moindre digestibilité iléale, de l'azote en particulier. Cet effet dépressif pourrait du reste relever autant d'une authentique réduction de la digestibilité que d'une augmentation de l'azote endogène.

Ceci étant, il est évidemment difficile de comparer la dépréciation de la digestibilité iléale enregistrée ici, et les informations contradictoires, résumées par PEREZ

(1) Nous remercions très vivement $\mathbf{M}^{\text {me }}$ Christiane MERcier, Laboratoire de Biochimie et Technologie des Glucides (I.N.R.A.-Nantes) qui a bien voulu vérifier les dosages d'amidon et réaliser le dosage des glucides alcoolo-solubles. 
et al. (1981) qui témoignent selon le cas d'une amélioration ou d'une détérioration de la digestibilité fécale et de la fréquence des diarrhées. La variabilité de la qualité des maniocs est telle qu'une comparaison des digestibilités iléale et fécale n'est envisageable que pour une même matière première. Certes les observations rapportées. dans ce travail devront être confirmées sur un plus grand nombre d'animaux, et pour divers types de manioc tel que celui utilisé dans l'aliment standard (manioc de Thailande) dont la digestibilité n'a pas été mesurée ici compte tenu de la complexité de la formulation. Ceci étant, les observations relatives aux modifications de la motricité suggèrent que les conséquences de l'ingestion de manioc peuvent constituer un terrain favorable à l'éclosion d'une pathologie digestive par la prolifération d'agents pathogènes opportunistes. Le manioc a été un temps suspecté d'être le responsablc d'un syndrome d'abord dit "des fèces molles", et désormais désigné sous le nom de diarrhée récurrente. La responsabilité directe de cette matière première paraît devoir être écartée dans la mesure où nous n'avons enregistré aucun signe diarrhéique chez nos porcs, que ce soit avec le régime semi-purifié à 72 p. 100 de manioc ou avec le régime standard prélevé dans un élevage atteint de diarrhée récurrente. Mais les maniocs destinés à l'alimentation animale restent d'une qualité relativement irrégulière, notamment en ce qui concerne l'épluchage des racines d'une part, et la conservation durant le transport d'autre part. Ccci reste vrai même pour un produit considéré a priori comme de bonne qualité. Aussi, un aliment riche en manioc est-il certainement susceptible, seul ou en association avec d'autres facteurs tels que l'abreuvement, de créer des conditions favorables à l'apparition d'une diarrhée récurrente. On sait en effet (JESTIN, 1984) que celle-ci est expérimentalement reproductible et transmissible, associée à des virus et bactéries au niveau de l'intestin grêle et à des protozoaires au niveau du gros intestin, tous agents dont la pathogénicité s'exprime différemment selon l'aliment consommé.

\section{En conclusion}

L'incorporation de manioc dans l'alimentation du porc ne présente d'inconvénient à l'égard du fonctionnement de l'intestin grêle que dans la mesure où cette matière première reste qualitativement imparfaite. En effet, l'amidon du manioc est digéré de façon complète dans l'intestin grêle. Mais la présence des composés phénoliques et tannins, du fait du non épluchage des racines, explique probablement la forte réduction de la digestibilité iléale de la matière sèche et de l'azote. La présence de quantités non négligeables de sucres simples, issus pour l'essentiel d'une dégradation de l'amidon au cours d'une conservation imparfaite, peut être à l'origine d'un afflux d'eau ver's la lumière et par voie de conséquence d'une perturbation voire désorganisation de la motricité. Cette situation constitue un terrain potentiel-. lement favorable pour des agents pathogènes opportunistes.

\section{Summary}

Small intestine motility and ileal digestibility of a cassava diet in the pig

Four Large White castrated male pigs averaging $50 \mathrm{~kg}$ live weight were used to estimate the effects of incorporating cassava into the diet on the motility of the small intestine, 
on the one hand, and on the apparent digestibility in the distal small intestine, on the other hand. Two of the pigs fitted with chronical electrodes implanted at 7 sites along the small intestine, were used to study the motility pattern of myoelectric migrating complexes during ingestion of semi-purified diets including 72.4 p. 100 cassava meal or 68.3 p. 100 purified maize starch. Each diet contained 16 p. 100 total crude protein mainly supplied by fish meal (table 1). Parallel to that a commercial standard diet including 30 p. 100 cassava meal was tested in the same experimental conditions. The other two pigs subjected to an ileo-colic post-valvular fistulation, were used in a qualitative and quantitative study of digesta collected at the ileo-caeco-colic junction after intake of a semi-purified diet containing cassava meal. The results were compared to those previously obtained with a diet based on purified maize starch (DARCY, LAPLACE \& VILlIERS, 1981).

The amounts of fresh matter (fig. 1) and dry matter (fig. 2) collected in the distal small intestine were 2.5 and 1.7 , respectively larger with the semi-purified cassava diet than with the maize-starch diet. The dry matter content of digesta (fig. 3) averaged only 7 p. 100 for the cassava diet versus 11 p. 100 for the maize diet. The ileal apparent digestibility of starch was subtotal for the two diets while those of dry matter and nitrogen (table 2) were highly reduced ( 78 p. 100 for cassava versus 87 p. 100 for maize). The motility pattern of myoelectric complexes (tabl. 3, 4 and 5) was almost maintained with the semi-purified cassava diet. However, some important changes in the characteristics of the complexes may be directly related with the increase in the volume of the more fluid cassava digesta. The rise in the level of intake when feeding the standard diet aggravated these effects and led to a true disturbance of the motility pattern (fig. 4). These observations can be associated with the presence in cassava meal of almost $5 \mathrm{p}$. 100 simple carbohydrates liable to be responsible for the afflux of water towards the intestinal lumen and thus for the motility changes; furthermore, the presence of phenol compounds and tannins is most likely responsible for the reduced ileal digestibility of dry matter and nitrogen. These features in connection with the irregular qualitative characteristics of the raw materials may lead to pathological situations such as the recurrent diarrhea syndrome.

Key words : motility, small intestine, ileal digestibility, cassava, pig.

Reçu en juin 1984.

Accepté en août 1984.

\section{Références bibliographiques}

Aumaitre A., 1969. Valeur alimentaire du manioc et de différentes céréales dans les régimes de sevrage précoce du porcelet : utilisation digestive de l'aliment et effet sur la croissance des animaux. Ann. Zootech., 18, 385-398.

Bueno L., RuCKebusch Y., 1978. Migrating myoelectrical complexes : disruption, enhancement, and disorganization, 83-90. In : DuTHIE H.L., Gastrointestinal motility in health and disease, MTP Press Ltd., Lancaster.

Darcy B., Laplace J.P., Villiers P.A., 1980. Digestion dans l'intestin grêle chez le porc. 2. - Cinétique comparée de passage des digesta selon le mode de fistulation iléo-caecale ou iléo-colique post-valvulaire, dans diverses conditions d'alimentation. Ann. Zootech., 29, 147-177.

Darcy B., Laplace J.P., Villiers P.A., 1981. Digestion dans l'intestin grêle chez le porc. 4. - Cinétique de passage des digesta au niveau de la jonction iléo-caeco-colique et bilans de la digestion selon la nature de l'amidon et la source de protéines alimentaires. Ann. Zootech., 30, 31-62.

JESTIN A., 1984. Résumé des premières recherches entreprises sur le syndrôme de la diarrhée récurrente dans les porcheries d'engraissement. Rapport de la Station de Pathologie Porcine, Ploufragan.

LAPLACE J.P., 1978a. Organisation de la motricité de l'intestin grêle chez le porc et influence de l'alimentation. Ann. Zootech., 27, 377-408. 
LAPLACE J.P., 1978b. Observations préliminaires sur quelques effets du lactose chez le porc. Complexes myoélectriques de l'intestin grêle, motricité caecale et diarrhée. Ann. Zootech., 27, 495-517.

LAPLACE J.P., 1980. Stomach and small intestine motility in the pig : electromyography in nutritional studies, 24-47. In : Low A.G., PARTridge I.G., Current concepts of digestion and absorption in pigs, N.I.R.D. Press, Reading.

LaPlace J.P., Roman C., 1979. Activités de la musculature gastrointestinale et mouvements des contenus digestifs. Ann. Biol. Anim. Biochim. Biophys., 19, 849-879.

Latour A., 1973. Un dispositif simple d'analyse quantitative de l'électromyogramme intestinal chronique. Ann. Rech. Vet., 4, 347-353.

OKE O.L., 1978 ; Problems in the use of Cassava as animal feed. Anim. Feed Sci. Technol., 3, 345-380.

Perez J.M., Castaing J., Grosjean F., Chauvel J., Bourdon D., Leuillet M., 1981. Valeur énergétique de deux types de manioc et utilisation comparée dans les régimes du porc en croissance et du porcelet : Synthèse des résultats d'une étude concertée, I.N.R.A.-I.T.C.F.-I.T.P., 125-144. In : Journées Rech. Porcine en France, I.N.R.A.I.T.P. Ed., Paris.

Ruckebusch Y., Bueno L., 1976. The effect of feeding on the motility of the stomach and small intestine in the pig. Br. J. Nutr., 35, 397-405.

Thivend P., Mercier C., Guilbot A., 1972. Determination of starch with gluco-amylase, 100-105, In : WHISTLER R.L., "Methods in carbohydrate chemistry 》, vol. VI, Academic Press, New York and London. 